\title{
PROJETO DE CONTROLADORES PID ROBUSTOS VIA OTIMIZAÇÃO NUMÉRICA COM APLICAÇÃO NO CONTROLE DE NÍVEL
}

\author{
Leonardi $\mathrm{F}^{*}$, Aguiar $\mathrm{RA}^{2}$, Franco $\mathrm{IC}^{3}$ e Menezes $\mathrm{MR}^{3}$
}

\section{ROBUST PID CONTROLLER DESIGN VIA NUMERICAL OPTIMIZATION WITH APPLICATION TO LEVEL CONTROL}

\section{F. LEONARDI ${ }^{1, *}$, R. A. AGUIAR ${ }^{2}$, I. C. FRANCO ${ }^{3}$ e M. R. MENEZES ${ }^{3}$}

${ }^{1}$ Centro Universitário FEI, Departamento de Engenharia Mecânica, São Bernardo do Campo, São Paulo, Brasil

${ }^{2}$ Centro Universitário FEI, Departamento de Engenharia Elétrica, São Bernardo do Campo, São Paulo, Brasil

${ }^{3}$ Centro Universitário FEI, Departamento de Engenharia Química, São Bernardo do Campo, São Paulo, Brasil

${ }^{*}$ Corresponding author. Centro Universitário da FEI, Depto de Engenharia Mecânica, São Bernardo do Campo, São Paulo, Brasil, Phone: +55 11 4353-2900

e-mail addressl:fabrizio@fei.edu.br (F. Leonardi).

\section{A R T I C L E I N F O}

Article history:

Received 2019-10-01

Accepted 2019-12-20

Available online 2019-12-20

palavras-chave

Shooting

Controle ótimo

Controle PID

Controle Robusto

\section{keywords}

Shooting

Controle ótimo

Controle PID

Robust Control

\section{R E S U M O}

O estudo de sistemas dinâmicos é um problema frequente nas diferentes áreas da Engenharia. Quando as especificações de desempenho relacionadas a esses tipos de sistemas são rigorosas, normalmente se utilizam sistemas de controle em malha fechada para garantir o desempenho esperado, mesmo na presença de erros de modelagem, ou seja, robustos às incertezas. O presente trabalho propõe a aplicação da técnica de shooting de otimização como alternativa para o projeto de controladores PID robustos, sendo aplicada a uma planta de controle de nível. Pelos resultados experimentais observados, pode-se concluir que a técnica pode conferir bom desempenho de regime permanente e transitório, avaliados pelo erro estacionário, tempo de acomodação e overshoot mesmo na presença de incerteza no valor da constante de tempo do modelo da planta.

A B S T R A C T

The study of dynamic systems is a frequent problem in different areas of engineering. Whenever the performance specifications related to these types of systems are strict, closed loop control systems are normally used to ensure expected performance even in the presence of modeling errors, ie robust to uncertainties. The present work proposes the application of the optimization shooting technique as an alternative for the design of robust PID controllers, being applied to a level control plant. From the experimental results observed, it can be concluded that the technique can give good performance of steady and transient state, evaluated by steady state error, settling time and overshoot, even in the presence of uncertainty in the value of the time constant of the plant model. 


\section{INTRODUÇ̃̃̃O}

Os sistemas do mundo real são inerentemente de natureza não linear, ao menos quando considerados em ampla faixa de operação (Liu et al, 2015). Entretanto, é comum utilizar-se de processos de linearização para viabilizar o projeto de controladores lineares, uma vez que os sistemas de controle em malha fechada toleram erros de modelagem, mesmo que propositais (Ogata, 2003)

A linearização é um procedimento simples, porém geralmente perde as características originais dos sistemas não lineares e pode levar a uma imprecisão ou conclusões incorretas (Iqbal et al., 2017) limitando assim sua aplicação, principalmente se quando a planta é operada longe do ponto de operação onde foi feita a linearização. Assim, projetar um sistema de controle para sistemas não lineares é geralmente um trabalho desafiador. O projetista deve ser capaz de analisar os efeitos de não linearidades indesejadas no sistema a fim de melhorar o desempenho dinâmico (Astrom e Kumar, 2014).

Nos últimos anos, os controles aplicados a sistemas não lineares vêm crescendo de forma exponencial, em particular em sistemas mecânicos, sistemas elétricos e processos químicos e bioquímicos. As principais razões para o crescente interesse incluem melhorias de sistemas de controle, análise de não linearidade, necessidade de lidar com incertezas dos modelos dinâmicos e simplicidade do projeto (Iqbal et al., 2017).

Estudos de sistemas não lineares foram realizados amplamente, incluindo análise de estabilidade (Zhang et al., 2013; Zhang et al., 2008), projeto de controladores (Li e Wang, 2010; Xi et al., 2014) e problemas de sincronização (Wang e Song, 2009; Wang e He, 2008). Várias novas ideias, abordagens e resultados surgiram no campo de controle não linear sendo a estabilidade uma abordagem central (Liu et al., 2015).

Dentre as alternativas possíveis para o projeto de malhas de controle, podem-se citar técnicas de otimização, tais como shooting e de colocação direta. A técnica de shooting permite resolver numericamente um problema de controle ótimo por meio de integração implícita do modelo dinâmico. A integração é feita para se avaliar a função objetivo e as restrições do problema.

A técnica de colocação direta faz a integração explícita do modelo dinâmico em cada instante de discretização do problema (Betts, 2010). Embora a técnica de colocação direta tenha tipicamente menos problemas de convergência, o número de equações diferenciais e sua estrutura devem ser invariantes. Já no shooting não importa o número de equações diferenciais e sua estrutura, bastando que o método seja capaz de integrar as equações no horizonte temporal do problema. Nesse cenário pode-se considerar o shooting como uma técnica mais geral para o projeto de sistemas de controle.

O Objetivo deste trabalho é ilustrar o projeto ótimo de um controlador PID robusto usando a técnica de shooting para uma planta não linear por meio do modelo linearizado em torno de um ponto de operação. Para ilustrar a metodologia considerou-se um problema de controle de nível de um tanque em escala laboratorial por meio de uma bomba centrífuga e saída aberta para a atmosfera. A incerteza considerada está relacionada com a constante de tempo do modelo que pode variar dependendo do ponto de operação

Note que embora o escopo deste trabalho é discutir algumas vantagens da técnica de shooting da otimização como metodologia para projeto de controladores, ilustrando a metodologia com um caso particular, ele pode ser visto como uma alternativa de projeto para controladores de nível.

Várias técnicas já foram aplicadas para controle de nível, como pode ser visto em (Pratama et al., 2016), em que os autores propõem o uso de um sistema de controle tipo gain scheduling para sintonização do PID no controle de nível. Nessa técnica, os ganhos do PID se auto ajustam de acordo com a dinâmica da planta, o que exige a necessidade de conhecer, com muita profundidade, as possíveis mudanças na dinâmica da planta que se está controlando. Também, um controlador PID de ordem fracionária, em que o integrador e o derivativo podem ser de ordem fracionária, foi utilizado para controle de nível de um tanque esférico (Sundaravadivu et al., 2011).

\section{METODOLOGIA}

\subsection{Planta didática}

Para o presente estudo, foi utilizada uma bancada de testes para controle de nível (DIDATICONTROL). Esta é equipada com dois tanques, T1 e T2 (com área de seção transversal de 48,5 cm2 cada), conectados a um reservatório comum por meio de duas válvulas de operação manual, V1 e V2. Possui também duas bombas hidráulicas que podem ter sua rotação alterada por meio de um sinal $\operatorname{PWM}(\operatorname{Vmax}=5 \mathrm{~V})$ e são responsáveis por bombear o líquido do reservatório aos tanques T1 e T2, e dois sensores de nível piezoresistivos. Um esquema do sistema descrito pode ser visualizado na Figura 1.

As válvulas V3 e V4, também de operação manual, conectam os tanques T1 e T2 em diferentes níveis.

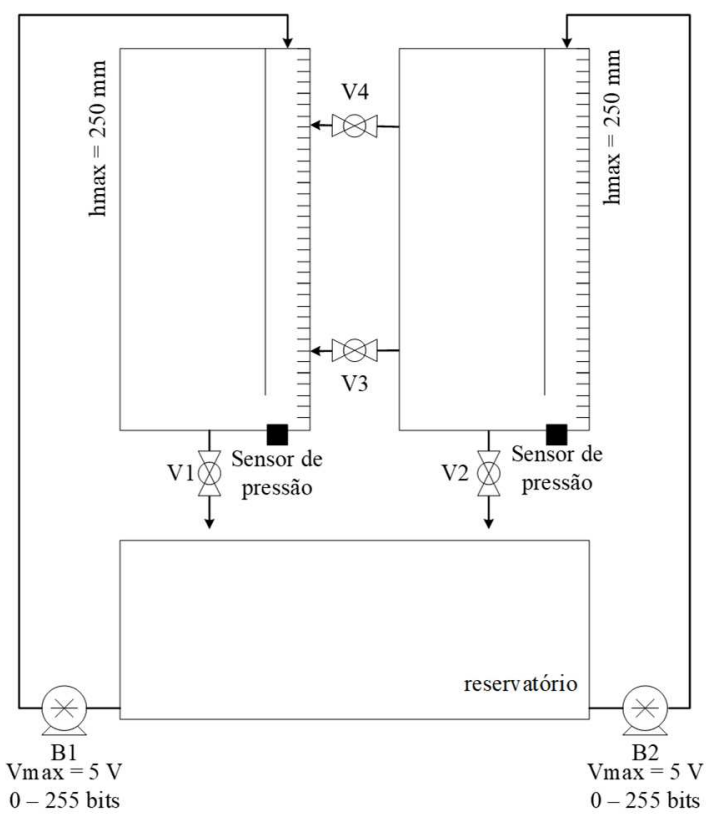




\section{Figura 1. Esquema da bancada didática para controle de nível utilizada e da placa de comunicação. \\ 2.2 Interface planta-arduino-simulink}

O sistema de controle foi desenvolvido no ambiente Simulink do Matlab ${ }^{\circledR}$ e para comunicação entre a planta e o simulink foi utilizado o add-on simulink support package for arduino hardware (disponível em www.mathworks.com). Os blocos utilizados assim como suas funções e principais parâmetros são apresentados na Tabela 1. A comunicação entre o Arduino e o simulink, quando o período de aquisição de dados for suficientemente pequeno, pode ser executada no modo normal, ou seja, sem a necessidade de realizar a compilação do código no Arduino. Caso o período de aquisição de dados não seja suficientemente pequeno, deve-se utilizar o modo external compilando o código para o Arduino. Nos experimentos deste trabalho utilizou-se um período de amostragem de $0,1 \mathrm{~s}$ em modo normal.

Tabela 1 - Blocos do add-on simulink support package for arduino hardware utilizados no desenvolvimento do sistema de controle.

\begin{tabular}{|c|c|c|}
\hline Bloco Simulink & Função & Parâmetros \\
\hline 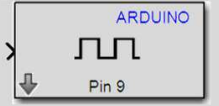 & $\begin{array}{ll}\text { Ligar/desligar } & \text { as } \\
\text { bombas } & \text { do } \\
\text { sistema } & \end{array}$ & $\begin{array}{l}\text { Pin number (número do } \\
\text { pino no qual está ligado } \\
\text { no Arduino) }\end{array}$ \\
\hline Arduino Analog Input & $\begin{array}{l}\text { Ler o valor do } \\
\text { sinal transmito do } \\
\text { sensor de nível } \\
\text { piezoresistivo }\end{array}$ & $\begin{array}{l}\text { Pin number (número do } \\
\text { pino no qual está ligado } \\
\text { no Arduino) } \\
\text { Sample time (taxa de }\end{array}$ \\
\hline$\frac{\underbrace{}_{\text {Pin: } 5} L}{\text { Arduino } P W M}$ & $\begin{array}{lr}\text { Aciona o } & \text { PWM } \\
\text { responsável } & \text { por } \\
\text { modulara } & \text { as } \\
\text { bombas } & \text { do } \\
\text { sistema } & \\
\end{array}$ & $\begin{array}{l}\text { Pin number (número do } \\
\text { pino no qual está ligado } \\
\text { no Arduino) }\end{array}$ \\
\hline
\end{tabular}

\subsection{Projeto do controlador}

Para projeto ótimo do controlador PID robusto propõe-se o uso da técnica shooting por meio da função fminunc do Matlab, codificando no Simulink o sistema a ser integrado numericamente (vide Figura 2). O modelo da planta, utilizado no projeto, foi obtido pela identificação do sistema por meio de um ensaio tipo degrau em torno do nível $100 \mathrm{~mm}$ e obteve-se um ganho de frequência nula $k_{g}=1,64$ e uma constante de tempo $\tau=88,7$. A incerteza considerada está relacionada com a constante de tempo do modelo que pode variar dependendo do ponto de operação. Considerou-se uma variação de $20 \%$ para mais e para menos em relação ao seu valor nominal.

Uma maneira de se formular o problema de robusto em face de incertezas é considerando todos os modelos representativos por meio um planejamento de experimento (DOE) para que as variações dos parâmetros sejam consideradas com relevância estatística. No presente caso só há um parâmetro incerto, então considerou-se o valor nominal $\tau$ e os valores extremos $1,2 \tau$ e $0.8 \tau$. Para que o controlador seja robusto, ele deve ser capaz de reduzir o erro do sistema de controle com qualquer uma das plantas. O diagrama de blocos da Figura 2 mostra como isso pode ser representado. A referência de entrada e os controladores PID são idênticos. Dessa forma há três sinais de erro distintos e três esforços de controle distintos que devem ser considerados simultaneamente no problema de otimização.

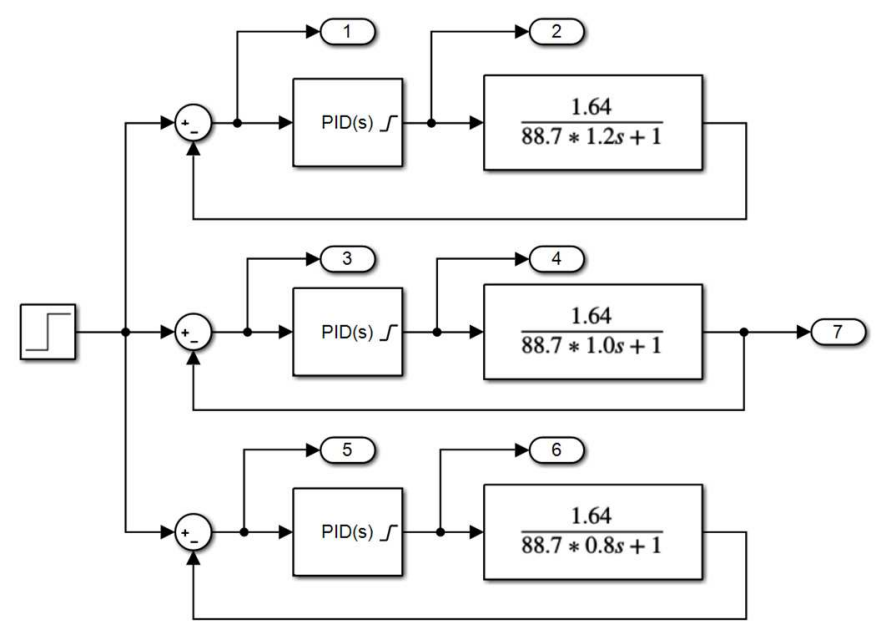

Figura 2. Diagrama de blocos Simulink utilizado na
otimização numérica.

O problema de controle robusto ótimo foi formulado como um problema de otimização sem restrições, embora a incorporação de restrições seja apenas função do solver utilizado. No caso sem restrições a função fminunc do Matlab é adequada e, no caso com restrições, pode-se utilizar por exemplo a função fmincon do Matlab. O problema de controle ótimo do tipo regulador linear quadrático pode ser colocado como (Eq. 1)

$\min _{k_{p}, k_{i}, k_{d}} J=\int_{0}^{t f}\left(Q \cdot \sum \mathrm{e}_{i}^{2}+R \cdot \sum \mathrm{u}_{i}^{2}\right) d t$

em que $\mathrm{e}_{i}(t)$ é o erro de acompanhamento do sinal de referência de cada sistema de controle, $\mathrm{u}_{i}(t)$ é o controle ou variável manipulada de cada sistema de controle, e $Q>0$ e $R>0$ são números reais que fazem a ponderação entre o desempenho do sistema de controle e o esforço de controle necessário. As variáveis do problema de otimização são os ganhos $\left\{k_{p}, k_{i}, k_{d}\right\}$ do controlador PID, que foram obtidos para valores dados de $Q$ e $R$, de forma que o esforço de controle fosse menor que a saturação para uma manobra de mudança do sinal de referência.

Note-se que as restrições dinâmicas dadas pelas equações diferenciais do sistema de controle foram implicitamente incorporadas na função objetivo, uma vez que para se determinar $J$ é necessário integrar as equações diferenciais relativas ao sistema de controle.

\section{RESULTADOS}

A técnica de shooting foi empregada para a solução do problema de controle ótimo robusto para um horizonte $t f=$ $50 s$ de forma a incluir a maior parte do transitório para uma manobra de mudança de setpoint de $10 \mathrm{~mm}$. Fixou-se $R=1 \mathrm{e}$ o valor $Q=100$ foi escolhido iterativamente de forma a causar uma variação máxima de 100 unidades no esforço de controle (PWM) quando a referência é alterada numa mudança de regime de $100 \mathrm{~mm}$ para $110 \mathrm{~mm}$. Os valores ótimos encontrados do PID foram: $k_{p}=10,09, k_{i}=0,083$ e $k_{d}=-0,103$.

Resultados experimentais foram obtidos aplicando-se o PID otimizado via shooting na planta real não linear apresentada na seção 2.1. Os resultados estão ilustrados pelos gráficos da figura $3 \mathrm{a}$. As primeiras quatro manobras de mudança de setpoint são de pequena amplitude e em torno do ponto de linearização (100 mm), fazendo com que a resposta do sistema não linear real 
seja bastante aproximada ao do modelo linearizado (veja detalhes do trecho na figura $3 \mathrm{~b}$ ). As demais manobras, à partir dos $880 \mathrm{~s}$, são de amplitudes elevadas e, como esperado, têm comportamento distinto ao do modelo linearizado. Note-se que nesses casos, mesmo com uma dinâmica bastante distinta, o sistema de controle consegue controlar a planta.

Como pode ser visto na figura $3 \mathrm{~b}$, foram feitas quatro manobras de mudança de regime: de $100 \mathrm{~mm}$ para $110 \mathrm{~mm}$, de $110 \mathrm{~mm}$ para $100 \mathrm{~mm}$, de $100 \mathrm{~mm}$ para $90 \mathrm{~mm}$ e de $90 \mathrm{~mm}$ para $100 \mathrm{~mm}$, o que permite destacar algumas características importantes de desempenho: como se vê, o tempo de acomodação é menor que 30 segundos e o erro de estado estacionário menor que $1 \%$ em todas a manobras executadas. Além disso, nota-se a ausência de overshoot em todos os casos.

Ressalta-se ainda, sem comparar o desempenho do sistema de controle aqui proposto com os sistemas de controle de nível supra mencionados, que os resultados obtidos neste trabalho são pertinentes e a técnica de projeto é interessante, pois permitiu conferir adequados erro estacionário, overshoot e tempo de acomodação, sem deixar de mencionar o pico máximo da variável manipulada (esforço de controle) cujo valor poderia ser facilmente inserido explicitamente como uma restrição do problema de otimização.
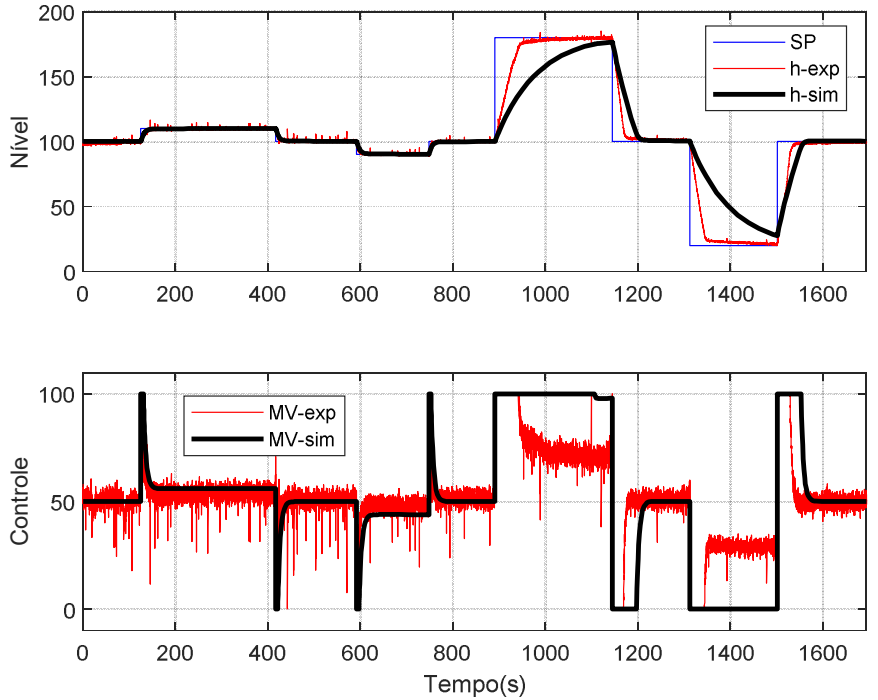

(a)
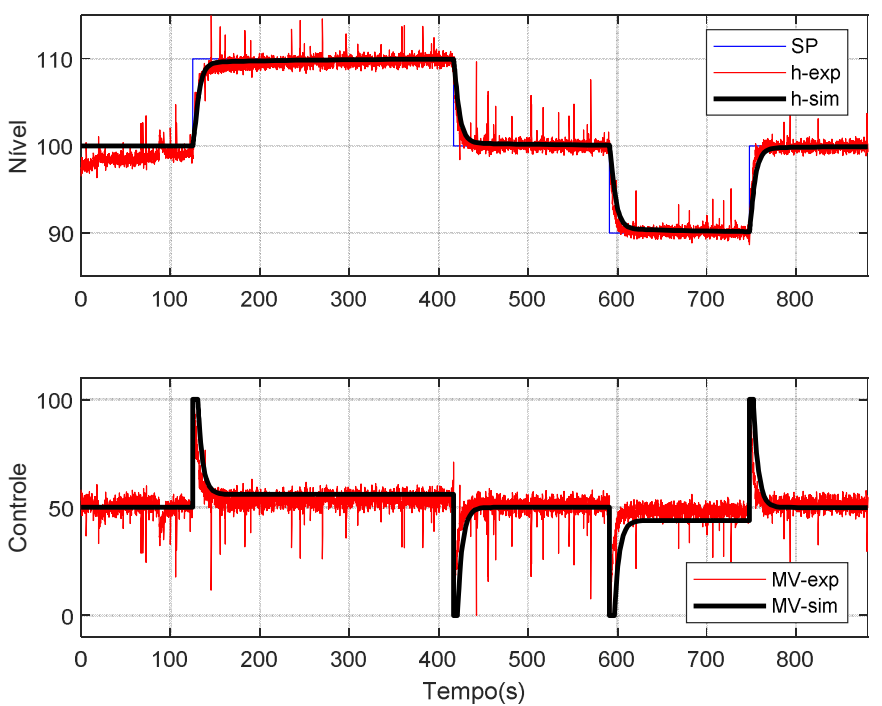

(b)

Figura 3. a) resultados experimentais; b) detalhe para as quatro primeiras manobras de mudança de setpoint.

\section{CONCLUSÕES}

O presente trabalho consistiu na aplicação da técnica de shooting de otimização para determinação dos parâmetros de um controlador PID robusto, visando seu desempenho dinâmico e de regime permanente, tanto no que diz respeito à resposta da variável controlada quanto ao esforço de controle aplicado à variável manipulada. Para atingir este objetivo, foi estudado um sistema não linear de controle de nível. Inicialmente, foram obtidos os parâmetros da planta mediante identificação o sistema, aplicando-se então a técnica de shooting para a solução de um problema de controle ótimo robusto em face de $20 \%$ de incerteza no valor da constante de tempo do modelo, e o desempenho do controlador assim sintonizado foi verificado, via simulação computacional, e também através da implementação em uma planta real, de maneira a ilustrar os resultados observados.

O controlador ajustado mediante a técnica proposta apresentou resultados bastante satisfatórios, quando observados os valores de overshoot, tempo de acomodação e erro estacionário, além de levar a um esforço de controle adequado, conduzindo a uma atuação suave, sem grandes oscilações ou saturação da variável manipulada, nos cenários estudados.

Como continuidade do trabalho, propõe-se utilizar as equações não lineares da planta no mesmo framework proposto para que manobras de grande amplitude possam ser explicitamente otimizadas.

\section{A G RA D E C I M E N T O S}

Os autores agradecem ao Centro Universitário FEI pelo apoio financeiro no desenvolvimento do trabalho.

\section{R E F E R E N C E S}

ASTROM, K. J.; KUMAR, P. R. Control: a perspective. Automatica, 50(1), 2-43,2014.

BETTS, J.T. Practical Methods for Optimal Control and Estimation Using Nonlinear Programming. Society for Industrial e Applied Mathematics, jan. 2010. p. 442.

IQBAL, J.; ULLAH, M.; KHAN, S. G; KHELIFA, B.; ĆUKOVIĆ, S. Nonlinear control systems - A brief overview of historical and recent advances. Nonlinear Eng. 6(4), 301-312, 2017.

LIN, D.; WANG, X. Observer-based decentralized fuzzy neural sliding mode control for interconnected unknown chaotic systems via network structure. Fuzzy Sets Syst. 161, 2066-2080, 2010.

LIU, Z.; HUANG, D.; XING, Y.; ZHANG, C.; WU, Z.; JI, X. New Trends in Nonlinear Control Systems and Applications. Abstract Appl. Anal. Article ID 637632, 2 pages, 2015.

OGATA, K. Engenharia de Controle Moderno. São Paulo: Pearson Education do Brasil, 2003.

PRATAMA, S. C.; SUSANTO, E.; WIBOWO, A. S. Design and Implementation of Water Level Control Using Gain Scheduling PID Back Calculation Integrator Anti 
Windup. The IEEE International Conference on Control, Electronics, Renewable Energy and Communications, 2016.

SUNDARAVADIVU, K.; ARUN, B.; SARAVANAN, K. Design of Fractional Order PID Controller for Liquid Level Control of Spherical Tank. IEEE International Conference on Control System, Computing and Engineering, 2011.

XIE, X.; YUE, D.; MA, T.; ZHU, X. Further studies on control synthesis of discrete-time $\mathrm{T}-\mathrm{S}$ fuzzy systems via augmented multi-indexed matrix approach. IEEE Trans. Cybern. 44, 2784-2791, 2014.

WANG, X.; HE, Y. Projective synchronization of fractional order chaotic system based on linear separation. Phys.
Lett. A 372, 435-441, 2008.

WANG, X.; SONG, J. Synchronization of the fractional order hyperchaos Lorenz systems with activation feedback control, Commun. Nonlinear Sci. Numer. Simul. 14, 3351-3357, 2009.

ZHANG, H.; YANG, F.; LIU, X.; ZHANG, Q. Stability analysis for neural networks with time-varying delay based on quadratic convex combination. IEEE Trans. Neural Netw. Learn. Syst. 24, 513-521, 2013.

ZHANG, H.; WANG, Z.; LIU, D. Global asymptotic stability of recurrent neural networks with multiple time-varying delays. IEEE Trans. Neural Netw. 19, 855-873, 2008 